\title{
Gestión de la emoción en la comunicación mediada por ordenador (CMO)
}

\author{
Beatriz PeÑA AcuÑA \\ Universidad Católica San Antonio de Murcia (UCAM) \\ bpena@ucam.edu \\ Mónica Rubio VegA \\ Universidad Católica San Antonio de Murcia (UCAM) \\ mrubiovega@yahoo.es
}

Recibido: $23 / 11 / 2012$

Aceptado: 23/01/2013

\section{Resumen}

Existe una concordancia entre diversos investigadores en defender que la comunicación emocional se da tanto en la relación presencial como en la Comunicación Mediada por Ordenador (CMO), de la misma manera en que están de acuerdo en afirmar que esta comunicación se produce con características distintas en ambas situaciones. Los estudios que analizan estas diferencias en el entorno investigador español son pocos y muy recientes, pero ya apuntan una serie de singularidades. En este estudio, se ha planteado analizar la expresión de las emociones en la $\mathrm{CMO}$ a partir de una encuesta realizada entre universitarios nativos digitales, que producen su comunicación emocional de un modo natural tanto de forma presencial como a través del ordenador.

Palabras clave: Internet, redes sociales, emoción, nativo virtual, comunicación mediada por ordenador

\section{Management of emotion in computer-mediated communication (CMC)}

\begin{abstract}
Concordance exists among some researchers who argue that the emotional communication occurs both in the face and in Computer-Mediated Communication (CMC), in the same way that they agree that this communication occurs with different characteristics in both situations. Studies examining these differences in the Spanish research environment are few and very recent, but already suggest a series of singularities. In this study, we analyze the expression of emotions in CMC from a survey of digital native university students that produce their naturally emotional communication both in person and through computer.
\end{abstract}

Keywords: Internet, social networks, emotion, native virtual, computer-mediated communication

\section{Referencia normalizada}

PEÑA ACUÑA, Beatriz y RUBIO VEGA, Mónica (2013): "Gestión de la emoción en la comunicación mediada por ordenador (CMO)". Estudios sobre el mensaje periodístico. Vol. 19. Núm. especial abril, págs.: 905-913. Madrid, Servicio de Publicaciones de la Universidad Complutense.

Sumario: 1. Introducción. 2. Metodología. 3. Desarrollo. 4. Conclusiones. 5. Referencias bibliográficas.

\section{Introducción}

Múltiples emociones circulan por la red: miedo, ira, alegría, sorpresa, tristeza y las derivadas de estas. Diversos autores, como Roser Beneito, Joaquín García Carrasco, Adam N. Joinson o Nicole Etchevers Goijberg, coinciden en esta afirmación. También apuntan que esta es una investigación incipiente en la que queda mucho camino por recorrer, como destacan, por ejemplo, Beneito o Etchevers. La duda en el entorno científico es si estas emociones se comunican con la misma eficacia en la Comunica- 
ción Mediada por Ordenador (CMO) que en la comunicación presencial tradicional y cuáles son sus singularidades. Para investigar estas cuestiones se acota el estudio a aquella CMO en la que sólo existe el texto y, por tanto, se excluye el uso de la cámara web. Para el análisis, se ha realizado una encuesta inspirada en el modelo de Beneito, entre universitarios nacidos entre 1990-1991, es decir, nativos digitales: "menores de 30 años, que han crecido con la tecnología y, por lo tanto, tienen una habilidad innata en el lenguaje y en el entorno digital", como exponen García y otros (García et al, 2007).

Contextualizando el origen de Internet, Arpanet nació en 1969. Adel apunta también que "El último "boom" de la Internet ha sido el World Wide Web (WWW)" (Adel, 1995: web) en 1990. El cariz psicosocial de la WWW estuvo muy presente en la mente de su creador Tim Berners-Lee: “[...] la génesis de la Web ha tenido en cuenta el parámetro psicosocial, la interconexión humana y la comunicación universal a través de vivencias personales". (Berners-Lee, 2000: web). Además, entre los recursos permitidos por Internet destacamos las redes sociales, uno de los servicios que permiten un mayor grado de comunicación entre usuarios. Se estima el año 2004 como el de su nacimiento (Fernández, 2008: web). En 2004, nuestros entrevistados tenían 13-14 años; una edad a la que hoy, los adolescentes manejan con soltura sus propios perfiles en la red. El estudio del AIMC del 2012 expone el uso frecuente de Internet que ya hacen los niños en España y el uso casi generalizado de los preadolescentes (AIMC, 2012: 3-4).

La comunicación emocional en Internet se da también en el correo electrónico, los chats, los móviles a través del What's App; y en el ordenador, en el móvil, el tablet... y otros servicios y dispositivos, y nos atrevemos a asegurar que se seguirá dando en servicios y dispositivos que aún no conocemos. Suscribimos la definición de Santoro (1995) de CMO (Computer Mediated Comunication, CMC, en inglés) como "extenso conjunto de funciones en las que el ordenador es utilizado para apoyar la comunicación humana" y de la que dice, y es parte que nos interesa, que "en su sentido más amplio puede abarcar virtualmente todas las utilidades informáticas". Esta y otras definiciones al respecto están recogidas en el trabajo de Perera Rodríguez (Perera y Torres, 2005: pág. 3-4). En esta línea se expresan Edgar Gómez y Alma Galindo cuando señalan que la $\mathrm{CMO}$ es "quizá una de las áreas más conocidas de Internet y una de las más utilizadas en forma de chats, mensajeros, grupos de noticias y, por supuesto, el correo electrónico" (Gómez y Galindo, 2005: web).

Diremos, además, como fundamento teórico psicosocial, que la conciencia sobre las reacciones físicas corporales propias es un aprendizaje de lo consciente sobre lo físico o corporal, lo que se dice, cómo conocer el cuerpo, reconocer los síntomas, etc. En otro nivel de aprendizaje encontramos lo que se denomina "inteligencia emocional" (Goleman, 1995) que consiste en la identificación con mayor grado de consciencia por parte de lo racional de la emoción motor de la propia reacción. Esta instrucción la han desarrollado aquellas personas que tienen mayor índice de conocimiento sobre sus emociones y sentimientos, los que tienen más desarrollada la inteligencia emocional y, por eso, son capaces de entender bien la de los demás, y estos acuden buscando ayuda y consejo de ellos. Suelen, además, saber gestionar bien sus emociones y no se dejan atrapar por ellas (Peña, 2011, 21). Ervin Goffman (1993) 
añade el hecho del posicionamiento debido a la aceptación y dramatización de roles sociales por convención social.

\section{Metodología}

La encuesta se realizó a 57 alumnos, estudiantes del Grado de Magisterio Infantil, de $3^{\circ}$ y $4^{\circ}$ Curso de la UCAM en Murcia, España. Con un promedio de 20-21 años, 55 eran mujeres y 2 hombres. El resultado podríamos denominarlo de género, pero en futuras investigaciones esperamos compensarlo con un número similar de hombres y añadir datos cualitativos de este género. Esta herramienta ha consistido en un cuestionario de catorce preguntas con respuestas múltiples de corte cuantitativa.

Algunas cuestiones se plantean comparativamente entre la comunicación presencial y la $\mathrm{CMO}$, como la eficacia y fiabilidad con la que se transmiten las emociones, la aparición de errores en la transmisión, la cantidad de reacciones físicas y emocionales, su intensidad, la estimación sobre la recepción de emociones y dónde reprimen más éstas. Otras investigan especialmente la CMO: la satisfacción con los recursos, el relato de emociones menores y profundas o la preferencia y los fines de uso de recursos expresivos.

\section{Desarrollo}

Expondremos los resultados más relevantes. De estas catorce preguntas, ocho han mostrado una tendencia más definida, o sea, un porcentaje superior al $70 \%$ de los entrevistados se decanta por la misma respuesta. En la primera pregunta se consulta a los encuestados sobre su percepción de la claridad (eficacia y fiabilidad) con la que se transmiten las emociones en la CMO frente a la comunicación presencial. El 98'2\% de los encuestados considera que la CMO no permite la misma claridad, y que la comunicación cara a cara es mejor situación para transmitir las emociones. Por lo tanto, entendemos que asumen la existencia de singularidades que diferencian cada una de las situaciones.

En la segunda pregunta, que se fija en la recepción de las emociones por parte del interlocutor virtual, los encuestados creen también, con un 98 ' $2 \%$, que sus interlocutores perciben con más claridad las emociones de una conversación presencial.

En la tercera cuestión, sobre si el entrevistado asume la posibilidad de que se produzcan errores en la transmisión de las emociones en la CMO, el 91'2\% opina que puede haber más errores en la CMO. En concordancia con la pregunta anterior, reflejan ser conscientes de que en la CMO se transmiten errores de comunicación al no haber ningún alumno que escoja la posibilidad de que no los haya. En una pregunta relacionada con ésta, y en correspondencia con ella, los encuestados consideran que sus interlocutores virtuales son también conscientes de que la CMO tiene características distintas a la comunicación presencial, ya que ninguno escoge que pueda darse la misma posibilidad de error en ambas situaciones. De hecho, un 63 ' $1 \%$ opina que sus interlocutores estiman que hay más errores en la CMO; mientras que un $14 \%$ cree, al contrario, que puede haber más errores en la comunicación dialógica, un dato que sobrepasa por mucho los resultados obtenidos en la pregunta anterior (que se quedaba en un anecdótico $1^{\prime} 75 \%$ ). También difiere, en este caso, que hasta un $21 \%$ cree que 
su interlocutor se comunica en la CMO sin tener en cuenta esta posibilidad de error.

En la cuarta cuestión se indaga sobre la cantidad de veces en que el entrevistado dice experimentar reacciones físicas durante sus conversaciones, tales como sudoración, vacilación o sonrojo. El 82`4\% de los participantes apunta que se producen en mayor cantidad en la comunicación cara a cara. A mucha distancia, con un $14 \%$, se sitúan quienes que consideran que se producen en cantidades similares. De forma excepcional, un 3'5\% dice sentirlas en más cantidad en la CMO. En otra pregunta paralela a la anterior, sobre la cantidad de veces en que el entrevistado dice experimentar, en este caso, reacciones emocionales, tales como pena, ansiedad y similares, la respuesta mayoritaria alcanza altas cotas, pero no con tanta diferencia (no superior al $70 \%$ ). Así, el $68^{\prime} 4 \%$ de los encuestados se decanta porque experimentan mayor cantidad de reacciones emocionales en la comunicación cara a cara (frente al $82 \%$ descrito en el caso de las reacciones físicas); mientras que la cifra de quienes dicen experimentarlas en cantidades similares en ambas situaciones alcanza el 28\% (frente al $14 \%$ del caso anterior). Por contra, se mantiene el 3'5\% de quienes dicen sentirlas en más cantidad en la CMO.

Igualmente, acerca de la pregunta quinta, referida a la intensidad de las expresiones emocionales como pena o ansiedad, los entrevistados indican en un $75^{\prime} 4 \%$ que se producen con mayor intensidad en la comunicación presencial. A distancia, con un $15^{\prime} 7 \%$, se agrupan quienes defienden que la intensidad es similar en ambos medios. Un $5 \%$ afirma que viven las expresiones emocionales con mayor intensidad en la CMO.

$\mathrm{Al}$ analizar la sexta pregunta, referida a la intensidad de las reacciones físicas como la sudoración, vacilación o sonrojo, vemos que el 73'6\% opina que esta intensidad es mayor en la comunicación cara a cara, un $17^{\prime} 5 \%$ defiende que siente una intensidad similar, y el 5'2\% dice experimentar más intensidad en sus reacciones físicas en la CMO.

Pasamos ahora a dos cuestiones que presentan la citada tendencia superior al 70\% una vez que se agrupan las respuestas. Así, en séptimo lugar incluimos la pregunta que aborda el uso de los recursos de la CMO para contar "emociones profundas", tipo: "Estoy muy triste porque he perdido un familiar" o "He roto con mi novio/a". En este caso, el uso habitual es del 1'7\% de los encuestados, mientras que lo usan ocasionalmente un $12{ }^{\prime} 2 \%$. Es decir, un $13{ }^{\prime} \% \%$ apuestan por comunicar estas situaciones a través de la CMO. Por contra, las opciones 'casi nunca', con un 19'29\%, y 'nunca', con un $66^{\prime} 6 \%$, alcanzan en conjunto un $85^{\prime} 9 \%$, de notoria tendencia. La pregunta antagonista a esta interroga sobre el uso de los recursos de la CMO para contar emociones con menor profundidad, que denominamos como "emociones menores", tipo: "Hoy estoy contenta/o", "Qué asco de día" o "Buen sábado a todos" (expresiones extraídas de la Red). Un 17 ' $5 \%$ responde que lo hace a menudo, frente a un $35 \%$ que lo hace ocasionalmente. Sin embargo, la opción 'casi nunca' aparece un 31 ' $5 \%$ y el $15^{\prime} 7 \%$ no lo ha hecho nunca. Así, observamos un reparto más equilibrado, pues un $52^{\prime} 6 \%$ de los encuestados se muestra favorable a su uso, y un $47^{\prime} 3 \%$ no tanto.

Por último, se planteó una pregunta global a los participantes sobre su satisfacción con los recursos que proporciona la $\mathrm{CMO}$ para transmitir emociones. Está totalmente 
satisfecho con ello un $8^{\prime} 7 \%$. Entre quienes no tienen una satisfacción total se halla el 43 ' $8 \%$ que se mostró bastante satisfecho, aunque piense que la CMO no es tan eficaz como la comunicación directa, y un $42^{\prime} 1 \%$ de quienes sí estaban satisfechos, pero menos que en comparación con el modo presencial (por cualquier motivo). Es decir, un $85^{\circ} 9 \%$ se muestra satisfecho con la $\mathrm{CMO}$, pero, en cualquier caso, lo está más con la comunicación presencial. Más aún, sumando estos tres grupos, un 94'7\% se muestra de una u otra forma satisfecho con la CMO. Un 5'2\% de los encuestados dice no estar satisfecho.

Hasta aquí, se han revisado once respuestas: las de las ocho preguntas que mostraban tendencia, más tres preguntas complementarias a estos datos, para su mejor comprensión. Veamos ahora las tres cuestiones restantes. Una de ellas investiga cuándo reprime más el usuario sus impulsos y tiene más autocontrol sobre acciones como insultar o escribir frases agresivas, valentonas, tiernas o sentimentales. Un 14\% indica que se reprime más en la CMO; un $28 \%$ se reprime más en la comunicación directa; y un $57^{\prime} 8 \%$ dice manejar su autocontrol sin tener en cuenta la situación comunicacional.

Por otra parte, para expresar las emociones, los recursos que más utilizan son, por orden de uso, los emoticones, con un 30' $6 \%$ de preferencia; las repeticiones de letras, con un $23{ }^{\prime} 1 \%$; y las interjecciones, con un $21{ }^{\prime} 2 \%$. Todo ello, frente a las frases explícitas (13’7\%) o el uso de mayúsculas (11'2\%). Acerca de en qué recursos recae su primera intención de uso, los usuarios también se decantan por los emoticones; pero seguido de las interjecciones y las repeticiones de letras.

$\mathrm{Y}$, finalmente, se preguntó a los usuarios sobre los fines de uso de estos recursos. Un $43{ }^{\prime} 5 \%$ indica que los usa para añadir más expresión a su escrito. Un 29\%, para aclarar alguna situación. Y un 23 ' $9 \%$ dice usarlos porque les divierte. A su vez, un $2{ }^{\prime} 5 \%$ reconoce no usarlos, y un $0{ }^{\prime} 8 \%$ dice hacerlo por imitación social.

\section{Conclusiones}

La encuesta realizada refleja que los internautas entrevistados, que por su edad y formación se manejan en la red de forma natural, consideran que la CMO no permite la misma claridad en la expresión de sus emociones que la tradicional comunicación presencial, tanto para la emisión como para la recepción de la comunicación; y entienden que la relación presencial proporciona una mejor situación comunicacional para transmitir las emociones. De esta forma, suponemos que estos usuarios no buscan en Internet un medio que supla sus relaciones presenciales, sino que las amplíe.

Consideran también que puede haber más errores de emisión de las emociones en la CMO que en la comunicación presencial. Suponemos que quizá esto se deba a que les falta el aporte de la Comunicación No Verbal, que incluye la kinesia (gestos de la cara, posición del cuerpo...), los elementos paralingüísticos (tono de voz, entonación...) y la proxémica (distancias...), lo que a su vez pueda conllevar una menor empatía, facultad más intuitiva. De hecho, entendemos que todos los recursos que se usan en la $\mathrm{CMO}$, como los emoticones, las interjecciones, las letras repetidas, las mayúsculas o las frases explícitas, creadas o no para este entorno virtual, son una forma de emular la información que aporta la Comunicación No verbal y que se halla ausente en la CMO. 
Los entrevistados estiman también, en su mayoría, que el interlocutor es más consciente de los errores de recepción en la $\mathrm{CMO}$, pero, de forma sorprendente, este grupo es casi treinta puntos menor que cuando se actúa como emisor. Estos usuarios 'que faltan' se reparten entre una parte menor que piensa que el receptor en la CMO está menos alerta a los errores; $y$ otra parte que cree que su interlocutor actúa indiferentemente del medio en el que recibe el mensaje. Es curioso indicar que esta última opción no fue contemplada por ningún entrevistado como emisor. Es decir: a la hora de emitir son conscientes de las diferencias de cada medio, pero creen que sus interlocutores no lo son. En cuanto a quienes creen que sus interlocutores están menos alertas en la CMO, es probable que desconfíen de la recepción, ya que su interlocutor puede no tener en cuenta un error en la transmisión de la emoción. Esto estaría de acuerdo con el alto porcentaje que en una pregunta anterior, se decantaba porque el interlocutor recibe las emociones con la mayor claridad en la relación presencial.

Acerca de las reacciones que los usuarios experimentan en ambas situaciones comunicacionales, tanto físicas como emocionales, es singular observar cómo la referencia a la intensidad obtiene prácticamente los mismos porcentajes, mientras que las diferencias se observan cuando describen la cantidad de estas reacciones. Al respecto, los datos reflejan que sus expresiones emocionales y físicas se producen con mayor intensidad en la comunicación presencial. Suponemos que la intensidad de respuesta emocional en un coloquio presencial sea mayor debido a la presión social que exige un posicionamiento; mientras que la CMO permite un distanciamiento del interpelado y un encubrimiento de las propias emociones frente a su/s interlocutor/es. A su vez, las reacciones físicas son una respuesta automática inevitable en un diálogo presencial en el que se da una plena exposición social. Cabe destacar que existe un grupo menor que dice tener la misma intensidad de reacciones en ambos modos de comunicar.

En cuanto a las diferencias en la cantidad en que se experimentan estas reacciones, los usuarios dicen percibir más cantidad de reacciones, tanto emocionales como físicas, en la CMO, pero el porcentaje es catorce puntos menos en lo que se refiere a las reacciones emocionales. En este sentido, también podemos suponer que la CMO permite ese distanciamiento, un tiempo de respuesta y un autocontrol; condición que no se da en un encuentro presencial, donde la presión social es mucho mayor. Así, estas circunstancias permitirían controlar las reacciones emocionales (pena, alegría...), cosa que habitualmente no podemos hacer con las reacciones físicas, o no tan fácilmente, ya que controlar un sonrojo, una sudoración o episodios similares requieren de un importante entrenamiento.

Por otra parte, un poco más de la mitad de los encuestados dice manifestar con naturalidad sus emociones tanto cara a cara como en la CMO. Y no solo eso, sino que un $28 \%$ dice reprimirse más en la comunicación presencial. Por otros estudios (Beneito: 2011), se esperaba que los alumnos indicaran un mayor autocontrol en la CMO, derivado del hecho de que la situación comunicacional en Internet permite un mayor distanciamiento con el interlocutor, tanto en el espacio -físicamente y sin imagen-, como en el tiempo -al poder retardar la respuesta- y, con ello, pensar más y poder controlar los impulsos y las reacciones físicas y emocionales. Sin embargo, es un grupo menor el que presenta mayor represión en la CMO. 
Todo tipo de información con contenido emocional se puede transmitir por Internet, sin embargo, las redes sociales reflejan un alto flujo de comentarios que hemos denominado "emociones menores", en las que se transmite un estado (espacio introducido, por ejemplo, en Facebook). Estos comentarios reflejan una emoción sin profundizar en ella. Así, nos ha llamado la atención que los entrevistados se reparten casi equitativamente entre los favorables y los desfavorables a su uso. Sin embargo, consideramos positivo que las redes sociales permitan esta expresión emocional global que, de alguna manera, consigue vincular y lograr un seguimiento emocional acerca de los internautas. Nos parece que, en este sentido, las redes sociales fomentan o permiten la expresión emocional de modo natural, como cultura. Creemos, incluso, que puede servir de vía de escape para emociones contenidas.

Sin embargo, al referirse a comunicaciones con mayor contenido emocional, los usuarios se posicionaron mayoritariamente contrarios a su uso, prefiriendo no comunicarlas a través de la CMO. Pensamos que se debe a que la manifestación de un estado global emocional no requiere explicación y de algún modo facilita compartir y fortalecer el vínculo emocional que ya se tiene entre interlocutores. En cambio, la expresión emocional profunda no es tan frecuente y requiere de un ambiente o situación favorable, mucha confianza e intimidad y un vínculo emocional mayor.

En cuanto al uso de recursos de expresión emocional, llama la atención que los recursos simbólicos (emoticones) sean más frecuentes frente a los recursos gráficos (repeticiones de letras, interjecciones, frases explícitas y mayúsculas). Elucubramos que pueda deberse a un uso más intuitivo y visual.

Es, además, interesante el resultado de que el principal fin de uso de los recursos de lenguaje de la CMO sea añadir más expresión para suplir todo el lenguaje corporal y los elementos paralingüísticos que faltan, como hemos comentado anteriormente. Asimismo, vemos que sigue habiendo conciencia en cuanto a la posibilidad de que se cometan errores, ya que casi un tercio de su uso se destina a aclarar situaciones. Por otro lado, es curioso que también los recursos de la CMO permitan, como característica, el uso lúdico.

Por último, constatamos que hay una tendencia marcada a mostrarse satisfecho con los recursos para la expresión emocional que proporciona la CMO. Ahora bien, entre los usuarios satisfechos se aprecia una tendencia tal que, si tienen que comparar, están más satisfechos con la comunicación cara a cara, cuestión que coincide con las anteriores conclusiones expuestas.

\section{Referencias bibliográficas}

ADEL, Jordi (1995): “Educación en la Internet”. Universitas Tarraconensis, serie IV, Vol. Extraordinari XX Setmana Pedagogica (ISSN 0211-3368), págs. 207-214. http://elbonia.cent.uji.es/jordi/wp-content/uploads/docs/tarragona.pdf [fecha de consulta: 20 octubre 2012]

BENEITO MONTAGUT, Roser (2011): “Comunicación interpersonal y emociones en internet”. Estudio que es parte del proyecto de investigación Everyday life on the Internet: interpersonal communications and emotions in web 2.0 / El dia a día en 
Internet: comunicación interpersonal y emociones en la web 2.0. http://mosaic.uoc.edu/2011/02/17/comunicacion-interpersonal-y-emociones-en-internet/. [fecha de consulta: 10 de junio de 2012]

BERNERS-LEE, Tim (2000): Tejiendo la Red. Madrid, Siglo XXI.

PEÑA ACUÑA, Beatriz (2010): "Entender el origen y la naturaleza de Internet", en Vivat Academia, $\mathrm{n}^{\circ}$ 110, marzo, pp. 1-10. http://www.ucm.es/info/vivataca/numeros/n110/Num110/PDFs/n110-Ensayo.pdf [fecha de consulta: 18 de octubre de 2012].

ETCHEVERS GOIJBERG Nicole (2005): “¿Dónde están las emociones en el Ciberespacio? Análisis de la situación actual". Revista Textos de la CiberSociedad, 5. Disponible en http://www.cibersociedad.net. [fecha de consulta: 10 de junio de 2012].

ETCHEVERS GOIJBERG; Nicole (2006): "Los nuevos códigos de la comunicación emocional utilizados en internet”. Revista Electrónica Teoría de la Educación. Educación y Cultura en la Sociedad de la Información. Vol. 7. Núm. 2. Diciembre. Disponible en http://www.usal.es/teoriaeducacion. [fecha de consulta: 10 de junio de 2012].

FERNÁNDEZ, Sonia (2008): "Redes sociales. Fenómeno pasajero o reflejo del nuevo internauta". Telos: cuadernos de comunicación e innovación. En http://sociedadinformacion.fundacion.telefonica.com/telos/articulocuaderno.asp@idarticulo\%3D11\&rev\%3D76.htm. [fecha de consulta: el 20 de octubre de 2012)

GARCÍA, Felipe; PORTILLO, Javier; ROMO, Jesús; BENITO, Manuel (2007): "Nativos digitales y modelos de aprendizaje". Actas IV Simposio Pluridisciplinar sobre Diseño, Evaluación y Desarrollo de Contenidos Educativos Reutilizables. Bilbao, en: http://spdece07.ehu.es/actas/Garcia.pdf [fecha de consulta: 15 de septiembre de 2012].

GARCÍA CARRASCO, Joaquín (2006): "Educación y cultura en la Sociedad de la Información". Revista Electrónica Teoría de la Educación. Vol. 7. Núm. 2. Diciembre. http://campus.usal.es/ teoriaeducacion/rev numero_07_02/n7_02_joaquin garcia.pdf. [fecha de consulta: 16 de junio de 2012].

GOFFMAN, Erving (1993): La presentación de la persona en la vida cotidiana. Buenos Aires, Amorrutu.

GOLEMAN, Daniel (1996): Inteligencia emocional. Barcelona, Kairós.

GÓMEZ, Edgar y GALINDO, Alma (2005): "Los estudios de la Comunicación Mediada por Computadora: una revisión y algunos apuntes". Razón y Palabra, abrilmayo Núm. 44. Disponible en: recinet.org. [fecha de consulta: 6 de agosto de 2012].

JOINSON, Adam N. (2003): "Understanding the psychology of Internet behaviour virtual worlds, real lives". Revista iberoamericana de educación a distancia. Vol. 6 N. ${ }^{\circ}$ (2003); disponible en: http://scholar.googleusercontent.com/scholar?q 
=cache:hwtMqHp_IdEJ:scholar.google.com/+emociones+Internet\&hl=es\&as_sdt $=0$. [fecha de consulta: 12 de junio de 2012].

PEÑA ACUÑA, Beatriz (coord., 2011): Desarrollo humano. Madrid, Visión libros.

PERERA RODRÍGUEZ, Víctor Hugo y TORRES GORDILLO, Juan Jesús (2005): "Una aproximación al estado actual de las investigaciones sobre la comunicación mediada por ordenador en el ámbito educativo", en el $V$ Congreso Internacional Virtual de Educación. Sevilla, Facultad de Ciencias, Universidad de Sevilla. Disponible en: www.cibereduca.com y en prometeo.us.es/idea/.../hugo/11.pdf. p.3-4. [Consultado el 6 de agosto de 2012]

\section{Beatriz PEÑA ACŨ̃NA}

Universidad Católica San Antonio de Murcia (UCAM)

Profesora contratada doctora

bpena@ucam.edu

\section{Mónica RUBIO VEGA}

Universidad Católica San Antonio de Murcia (UCAM)

Miembro del Grupo de Investigación Desarrollo Personal-UCAM

mrubiovega@yahoo.es 